\title{
9-Meric Peptide Analogs of Defensin-like Antimicrobial Peptide Coprisin with Potent Antibacterial Activities with Bacterial Sell Selectivites
}

\author{
Areum Shin, Eunjung Lee, Jin-Kyoung Kim, Jeong-Kyu Bang, ${ }^{\dagger}$ and Yangmee Kim* \\ Department of Bioscience and Biotechnology, Konkuk University, Seoul 143-701, Korea. *E-mail: ymkim@konkuk.ac.kr \\ ${ }^{\dagger}$ Division of Magnetic Resonance, Korea Basic Science Institute, Chung-Buk 363-883, Korea \\ Received June 16, 2014, Accepted July 19, 2014
}

\begin{abstract}
The 43-residue defensin-like peptide coprisin, which is isolated from dung bettle, Copris tripartitus, is a potent antimicrobial peptide. In our previous work, we determined the tertiary structure of coprisin and found that alpha helical region of coprisin from residue 19 to residue 30 is important for its antimicrobial activities. Here, we designed cop12mer and cop9mer analogs of coprisin based on the tertiary structure of coprisin. To investigate the relationship between hydrophobicity and antimicrobial activities and develop the potent peptide antibiotics, we designed cop9mer-1 with substitution of $\mathrm{His}^{2}$ with Trp in cop9mer. The results showed that cop9mer-1 has higher toxicities as well as improved antimicrobial activities compared to cop9mer. In order to reduce the toxicity of cop9mer-1, we designed cop9mer- 2 and cop9mer-3 with substitution of Cys ${ }^{3}$ with Lys or Ser. Substitution of $\mathrm{Cys}^{3}$ with these hydrophilic amino acids results in lower cytotoxicities compared to cop9mer-1. Cop9mer-2 with substitution of $\mathrm{Cys}^{3}$ with Lys in Cop9mer-1 showed high antibacterial activities against drug resistant bacteria without cytotoxicity. Antibiotic action of cop9mer-1 analog appears to involve permeabilization of the bacterial cell membrane while cop9mer-2 and cop9mer-3 may have different mechanism of action. These results imply that that optimum balance in hydrophobicity and hydrophilicity in these 9-meric peptides plays key roles in their antimicrobial activities as well as cytotoxicities.
\end{abstract}

Key Words : Coprisin, Antimicrobial peptide, Antibacterial activity, Cytotoxicity

\section{Introduction}

These days the emergence of antibiotic-resistant bacterial strains is needed for new antibiotic candidates. Antimicrobial peptides are thought as the alternative antibiotics. ${ }^{1}$ Antimicrobial peptides are found in a variety of species, including vertebrate, invertebrate and insects. ${ }^{2,3}$ These antimicrobial peptides play an essential role for their host immune systems. Although the detailed mechanism to kill invaded bacteria has not described yet, antimicrobial peptides seem to have the action mode of depolarization or permeabilization of bacteria cell membrane. ${ }^{1-5}$

More than 200 antimicrobial peptides isolated from insects were identified to date. Defensins are one of the antimicrobial peptide groups, which have conserved beta-sheet structure and intramolecular disulfide bonds. ${ }^{6-8}$ To date, defensins including HNP-1 to 5, drosomycin, sapecin and lucifensin were discovered from a variety of organisms. ${ }^{8-13}$ These defensins showed antimicrobial activities against gramnegative and gram-positive bacterial strains. ${ }^{6-13}$ Particularly, defensins from insects seems to be very active to various kinds of bacteria. Protaetiamycine, for example, having 43amino-acids antimicrobial peptide is one of defensin-like antimicrobial peptide which isolated form larvae of a beetle, Protaetia brevitarsis. ${ }^{14-16}$ In order to develop potent peptide antibiotics, we developed the 12-meric peptide which has the residue at alpha-helical region of protaetiamycine as well as its 9-meric derivative. ${ }^{14}$ To increased antifungal and antimicrobial activities, sequence of 9-meric analogs have been optimized with hydrophobicity and cationicity. ${ }^{14-16}$

Coprisin (VTCDVLSFEAKGIAVNHSACALHCIALRK KGGSCQNGVCVCRN-NH ${ }_{2}$ ) is a 43-residue defensin-like antimicrobial peptide which isolated from a Korean dung beetle, Copris tripartitus. ${ }^{17}$ In our previous study, coprisin shows the antimicrobial activities with low toxicities against mammalian cells and induces apoptosis against C. albicans. ${ }^{17,18}$ Coprisin also has anti-inflammatory activities in LPS-stimulated RAW 264.7. ${ }^{17}$ Three dimensional structure of coprisin has been determined using nuclear magnetic resonance spectroscopy and consists of two beta-sheets and one amphipathic alpha-helical structure linked by three disulfide bonds. ${ }^{17}$ Since the alpha-helical structure from Ala ${ }^{19}$ to $\mathrm{Lys}^{30}$ (ACALHCIALRKK-NH ${ }_{2}$ ) was revealed as the active domain for coprisin activities, ${ }^{18}$ we designed cop12mer with this active region in this study. The three residue truncated peptide (LHCIALRKK-NH ${ }_{2}$ ), called cop9mer, also designed to develop potent peptide antibiotics with short length. ${ }^{19}$ In this study, we focused on the short antimicrobial peptide, cop9mer and the balance between hydrophobicity and hydrophilicity on antimicrobial activities of peptides were examined. We designed 9-mer peptide analogs of cop9mer to increase antibacterial activity without cytotoxicity and investigated their antimicrobial activities as well as mechanism of action.

\section{Materials and Methods}

Peptide Synthesis. Coprisin analogs were synthesized by 
the standard Fmoc-based solid-phase method. The peptides were purified by reverse phase high-performance liquid column on a Vydac C18 column $(20 \times 250 \mathrm{~mm}$, Shim-pack $)$ using an appropriate $0-90 \%$ water/acetonitrile gradient in the presence of $0.05 \%$ trifluoroacetic acid. The final peptides have over $98 \%$ purity. The molecular mass of the purified peptides was determined by matrix-assisted laser-desorption ionization-time-of-flight mass spectrometry (Shimadzu, Japan).

Antimicrobial Activity. Escherichia coli (KCTC 1682), Salmonella typhimurium (KCTC 1926), Bacillus subtilis (KCTC 3068), and Staphylococcus aureus (KCTC 1621) were purchased from the Korean Collection for Type Cultures, Korea Research Institute of Bioscience and Biotechnology (Taejon, Korea). The Culture Collection of Antibiotic-Resistant Microbe (Seoul, Korea) supplied the isolates of multidrug-resistant Escherichia coli (CCARM 1238) multidrug-resistant Salmonella typhimurium (CCARM 8003) and methicilin-resistant Staphylococcus aureus (CCARM 3114). Minimal inhibitory concentrations (MICs) of peptides against bacteria were tested using a broth microdilution assay as described before. ${ }^{20}$ The MIC values were compared with those of melittin and coprisin. The lowest concentration of peptide that completely inhibited growth was defined as the MIC.

Hemolytic Activity. The hemolytic activity of peptide was performed using human red blood cells (hRBCs) as described before. ${ }^{21}$ The release of hemoglobin was monitored by measuring the absorbance of the supernatant at $405 \mathrm{~nm}$. No hemolysis (blank) and 100\% hemolysis controls consisted of hRBCs suspended in PBS and $0.1 \%$ Triton X-100, respectively. Percent hemolysis was calculated using the following equation,

Hemolysis $(\%)=\left[\left(\mathrm{OD}_{405 \mathrm{~nm}}\right.\right.$ sample $-\mathrm{OD}_{405 \mathrm{~nm}}$ zero lysis $)$ $/\left(\mathrm{OD}_{405 \mathrm{~nm}} 100 \%\right.$ lysis - $\mathrm{OD}_{405 \mathrm{~nm}}$ zero lysis $\left.)\right] \times 100$.

Calcein Leakage Assay. Calcein-cetrapped large unilamellar vesicles (LUVs) composed of egg yolk L- $\alpha$-phosphatidylcholine (EYPC)/egg yolk L- $\alpha$-phosphatidylglycerol (EYPG) (7:3, w/w), or egg yolk PC/cholesterol (CH) (10:1, $\mathrm{w} / \mathrm{w})$ were prepared as previously described. ${ }^{22}$ The leakage of calcein from LUVs was monitored by measuring fluorescence intensity using an excitation wavelength of $490 \mathrm{~nm}$ and an emission wavelength of $520 \mathrm{~nm}$. All fluorescence experiments were performed in quartz cuvettes with a $1-\mathrm{cm}$ path length using a model RF-5301PC spectrophotometer (Shimadzu, Kyoto, Japan). Vesicles dissolved in Tris buffer containing 10\% Triton X-100 (10 $\mu \mathrm{L}$ were used to establish $100 \%$ dye release). The percentage of dye leakage caused by peptides was calculated in the following equation, dye leakage $(\%)=100 \times\left(\mathrm{F}-\mathrm{F}_{0}\right) /\left(\mathrm{F}_{\mathrm{t}}-\mathrm{F}_{0}\right)$, where $\mathrm{F}$ is the fluorescence intensity of peptide-treated vesicles, and $F_{0}$ and $F_{t}$ are fluorescence intensities without the peptides and with Triton $\mathrm{X}-100$, respectively.

\section{Results and Discussion}

Activities of antimicrobial peptides are affected by a
Table 1. Amino acid sequences and properties of coprisin analogs

\begin{tabular}{cccc}
\hline Peptides & Amino acid sequence & $\begin{array}{c}\text { Net } \\
\text { Charge }^{a}\end{array}$ & Hydrophilicity $^{b}$ \\
\hline Cop12mer & ACALHCIALRKK-NH & 4 & 0.12 \\
Cop9mer & LHCIALRKK-NH & 4 & 0.38 \\
Cop9mer-1 & LWCIALRKK-NH $_{2}$ & 4 & 0.056 \\
Cop9mer-2 & LWKIALRKK-NH $_{2}$ & 5 & 0.5 \\
Cop9mer-3 & LWSIALRKK-NH $_{2}$ & 4 & 0.2 \\
\hline
\end{tabular}

${ }^{a}$ Net charge was calculated using sum of each of the amino acid charge at $\mathrm{pH}$ 7.0. ${ }^{b}$ Hydrophilicity is the total hydrophilicity (sum of the hydrophilicity indices of all the residues) divided by the number of residues, according to the Hopp and Woods index..$^{23}$

number of parameters, including net charge and hydrophobicity. In our previous study on protaetiamycin which is one of defensin-like antimicrobial peptide in insect, alpha helical region of the peptide which is consisted of 12 residues was important for its antimicrobial activities. ${ }^{14-16}$ Truncated 9-meric analogs remained antibacterial activities and we found that peptides with high hydrophobicity by substitution with Trp increases antimicrobial activities of peptides. ${ }^{15,16}$ It is well known that Trp interacts with bacterial cell membrane strongly and the aromatic ring inserted deep into the cell membrane. As listed in Table 1, coprisin analogs were synthesized based on the structure of coprisin (Figure 1). Cop12mer has been designed with the sequence in the active alpha helical region of coprisin from Ala ${ }^{19}$ to $\mathrm{Lys}^{30}$ (ACALHCIALRKK-NH ${ }_{2}$ ). The three residue truncated peptide (LHCIALRKK-NH $\mathrm{N}_{2}$ ), called cop9mer, also designed and synthesized. In order to increase the antibacterial activities of 9-meric analogs of coprisin, we designed Cop9mer-1 with substitution of Trp for $\mathrm{His}^{2}$ which has increased hydrophobicity and stronger interaction with cell membrane than cop9mer.

The antimicrobial activities were measured against the four standard bacterial strains, including two Gram-negative species (E. coli, S. typhimurium and) and two Gram-positive species (B. subtilis, and $S$. aureus). Antimicrobial activities of cop9mer-1 were compared with coprisin, cop 12 mer and

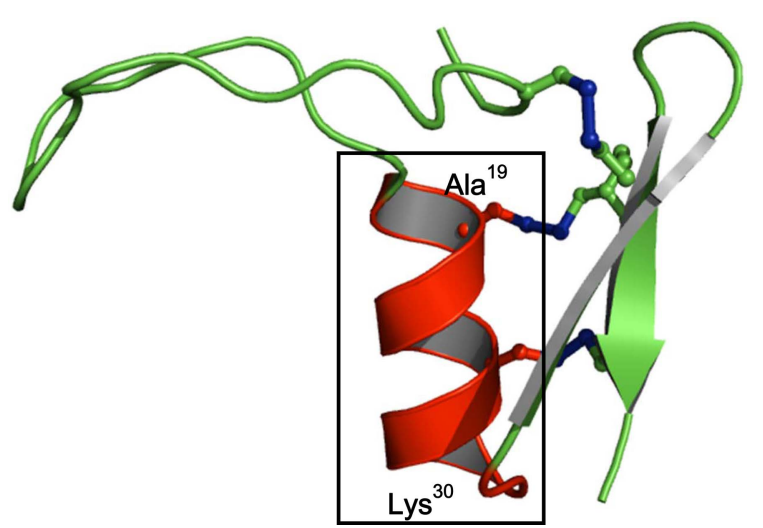

Figure 1. The tertiary structure of coprisin in DPC micelles at $\mathrm{pH}$ 4.3. (PDB ID : 2LN4) Active site including alpha helix from $\mathrm{Ala}^{19}$ to $\mathrm{Lys}^{30}$ in red is shown with box. 
Table 2. Antimicrobial activities of peptides against standard bacterial strains

\begin{tabular}{|c|c|c|c|c|c|c|c|c|}
\hline & Peptides & Coprisin & Cop12mer & Cop9mer & Cop9mer-1 & Cop9mer-2 & Cop9mer-3 & Melittin \\
\hline \multirow{4}{*}{$\begin{array}{l}\text { MIC } \\
(\mu \mathrm{M})\end{array}$} & E. coli & 0.5 & 16 & 16 & 2 & 2 & 4 & 1 \\
\hline & S. typhimurium & 2 & 32 & 32 & 8 & 4 & 32 & 8 \\
\hline & S. aureus & 2 & $>32$ & 32 & 8 & 16 & 16 & 2 \\
\hline & B. subtilis & 2 & $>32$ & 32 & 8 & 16 & 32 & 1 \\
\hline & $\mathrm{GM}^{a}$ & 1.6 & 24 & 28 & 6.5 & 9.5 & 21 & 3 \\
\hline & $\mathrm{MHC}^{b}$ & 200 & 200 & 200 & 25 & 200 & 200 & 0.38 \\
\hline Therap & ic index $(\mathrm{MHC} / \mathrm{GM})$ & 130 & 8.3 & 7.1 & 3.8 & 21 & 9.5 & 0.13 \\
\hline
\end{tabular}

${ }^{a}$ The geometric means (GM) of the MIC values from all four bacterial strains are shown. ${ }^{b}$ MHC is the minimal peptide concentration producing hemolysis. When no detectable hemolysis was observed at $100 \mu \mathrm{M}$, a value of $200 \mu \mathrm{M}$ was used to calculate the therapeutic index. ${ }^{c}$ The ratio of the MHC over the GM of MIC is shown. Larger values indicate greater cell selectivity.

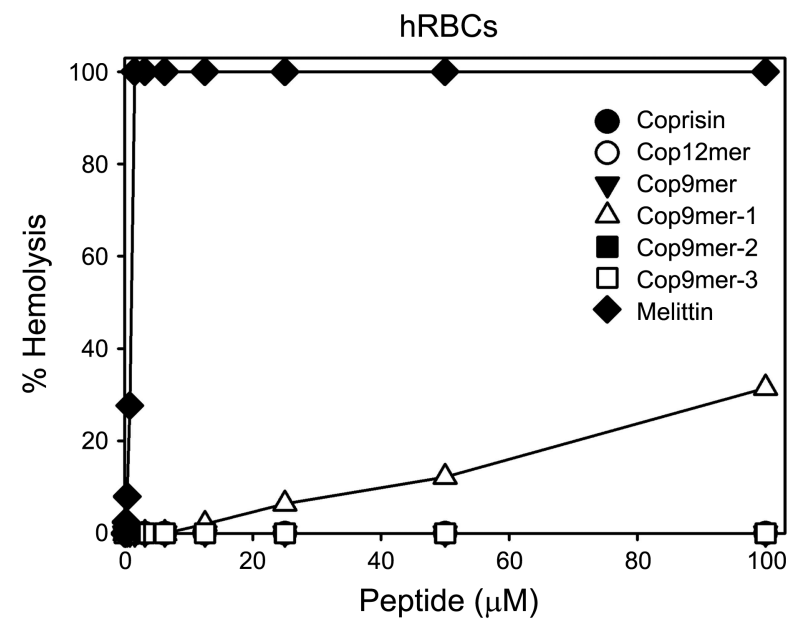

Figure 2. Dose-response curves of the hemolytic activity against human red blood cells for the antimicrobial peptides, coprisin, cop12mer and cop9mer analogs.

cop9mer. As listed on Table 2, Cop9mer-1 showed much higher antimicrobial activities than cop12mer and cop9mer. We next investigated hemolytic activity of cop9mer-1 against human red blood cells by measuring the extent of lysis of hemoglobin caused by the peptide. The results suggested that the cop9mer-1 is cytotoxic at high concentration and had $31 \%$ hemolytic activity at $100 \mu \mathrm{M}$ as shown in Figure 2.

To reduce cytoxicities against mammalian cells and maintain the antimicrobial activities of cop9mer-1, we designed two analogs with substitution of Lys or Ser for $\mathrm{Cys}^{3}$ in cop9mer-1 to increase hydrophilicity, called cop9mer-2 or cop9mer-3, respectively. As shown in hemolytic activity (Figure 2) data, cop9mer-2 and cop9mer-3 analogs reduced the cytoxicities against human red blood cells. Particularly, cop9mer-2 had higher antimicrobial activities against gramnegative bacteria than cop9mer-1. In order to develop potent therapeutic antibiotics, the peptide must have antimicrobial activities but no toxicity against mammalian cells. Therapeutic index in Table 2 shows the ratio of MHC to the average MIC of the peptides tested. The larger therapeutic index means higher cell selectivity. Cop9mer-2 has the largest therapeutic index value than cop9mer-1 and cop9mer-3. In Table 3, antimicrobial activities of coprisin analogs against antibioticresistant bacterial strains are listed and Cop9mer-2 has the highest antibacterial activities against gram-negative drug resistant bacteria among four 9-meric peptides.

To investigate the membrane-permeabilizing ability of cop9mer analogs, we monitored the release of the fluorescent marker, calcein, from LUVs with different composition. We employed zwitterionic LUVs composed of 10:1 (w/w) EYPC/CH which mimic components of mammalian cell membrane and negatively charged LUVs composed 7:3 (w/w) EYPC/EYPG which mimic components of the bacterial cell membrane. Non-cell-selective peptides bind and permeate into both of bacterial cell mimic phospholipid membrane which is negatively charged phospholipid membrane and mammalian cell mimic phospholipid membrane which is zwitterionic phospholipid membrane. However, most bacterial cell-selective peptides bind and permeate more efficiently into bacterial cell mimic phospholipid than into mammalian

Table 3. Antimicrobial activities of coprisin analogs against antibiotic-resistant bacterial strains

\begin{tabular}{ccccccc}
\hline & & Coprisin & Cop9mer-1 & Cop9mer-2 & Cop9mer-3 & Melittin \\
\hline \multirow{2}{*}{ MIC } & E. coli 1238(R) & 2 & 16 & 8 & 16 & 4 \\
$(\mu \mathrm{M})$ & S. typhimurium 8003(R) & 8 & 16 & 8 & $>32$ & 8 \\
& S. aureus 3114(R) & 2 & 16 & 32 & $>32$ & 1 \\
\hline $\mathrm{GM}^{a}$ & 4 & 16 & 16 & $>27$ & 4 \\
\multicolumn{2}{r}{ Therapeutic index } \\
& $\mathrm{MHC}^{b}$ & 200 & 25 & 200 & 200 & 0.38 \\
\hline
\end{tabular}

${ }^{a}$ The geometric means (GM) of the MIC values from all four bacterial strains are shown. ${ }^{b} \mathrm{MHC}$ is the minimal peptide concentration producing hemolysis. When no detectable hemolysis was observed at $100 \mu \mathrm{M}$, a value of $200 \mu \mathrm{M}$ was used to calculate the therapeutic index. ${ }^{c}$ The ratio of the MHC over the GM of MIC is shown. Larger values indicate greater cell selectivity. 


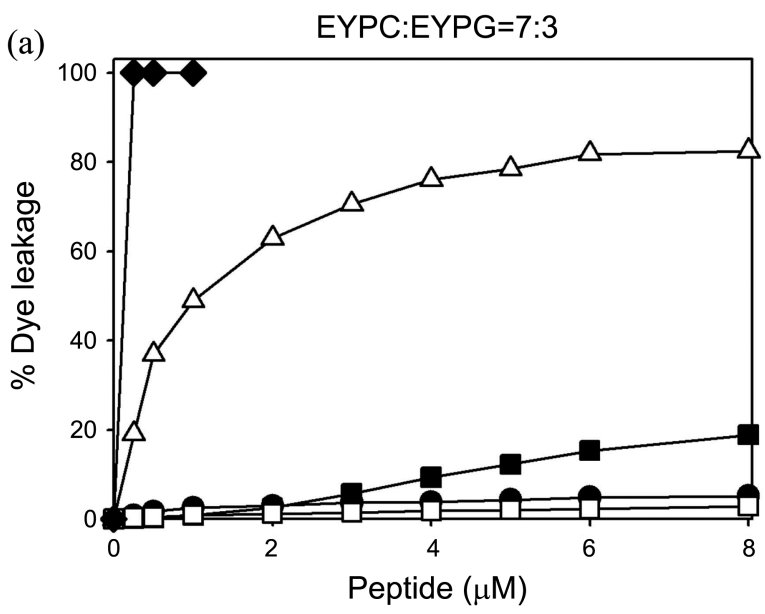

(b)

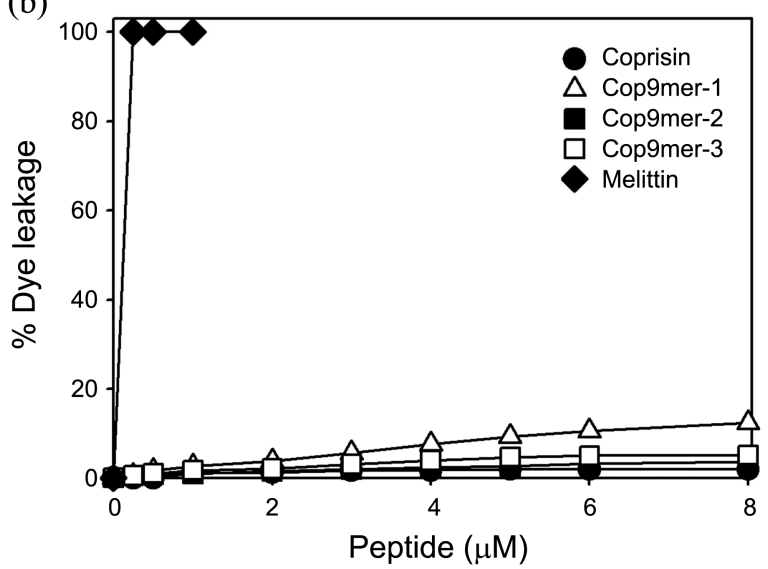

Figure 3. Dose-response curves of calcein leakage from (a) EYPC/EYPG (7:3, w/w) and (b) EYPC/CH LUVs (10:1, w/w) LUVs induced by the peptides. Calcein leakage was measured 2 min after adding the peptide. The $200 \mu \mathrm{M}$ lipid was used in the leakage experiments.

cell mimic phospholipid membrane. The dose-response curves of peptide-induced calcein release were represented in Figure 3. Cop9mer-1 permeated negatively charged vesicles much more effectively than zwitterionic vesicles, but cop9mer-2 and cop9mer-3 showed a little leakage from neutral charged vesicles, implying that these two peptides are not cytotoxic against mammalian cell. Interestingly, cop9mer-2 and cop9mer-3 showed a little leakage from negatively charged vesicles, too.

\section{Conclusion}

In this study, we attempted to design potent antimicrobial peptides with optimum balance between hydrophobicity and hydrophilicity. Cop9mer-1 with high hydrophobicity has high cytotoxicity as well as high antibacterial activities. Cop9mer-2 with higher cationicity retained high antimicrobial activities and showed low cytoxicities against human erythrocytes compared to Cop9mer-1. Cop9mer-3 with substitution of Cys with Ser in Cop9mer-1 showed less cyto- xicity as well as less antimicrobial activities compared to cop9mer-1. The release of the fluorescent marker from LUVs with different composition implies that cop9mer-1 has antimicrobial activity through permeabilization of the bacterial cell membrane while cop9mer-2 and cop9mer-3 does not target the bacterial cell membrane. The mechanism of action of cop9mer-2 and cop9mer-3 should be studied further. This study will be useful to design novel short antimicrobial peptide with high antimicrobial activities and no cytoxicities against mammalian cells.

Acknowledgments. This paper was supported by Konkuk University in 2013.

\section{References}

1. Hancock, R. E.; Sahl, H. G. Nat. Biotechnol. 2006, 24, 1551-1557.

2. Hancock, R. E.; Lehrer, R. Trends Biotechnol. 1998, 16, 82-88.

3. Zasloff, M. Nature 2002, 415, 389-395.

4. Yeaman, M. R.; Yount, N. Y. Pharmacol. Rev. 2003, 55, 27-55.

5. Nguyen, L. T.; Haney, E. F.; Vogel, H. J. Trends Biotechnol. 2011, $29,464-472$.

6. Selsted, M. E.; Szklarek, D.; Lehrer, R. I. Infect. Immun. 1984, 45, 150-154.

7. Selsted, M. E.; Szklarek, D.; Ganz, T.; Lehrer, R. I. Infect. Immun. 1985, 49, 202-206.

8. Wong, J. H.; Xia, L.; Ng, T. B. Curr. Protein Pept. Sci. 2007, 8, 446-459.

9. Chen, H.; Xu, Z.; Peng, L.; Fang, X.; Xin, X.; Xu, Z.; Chen, P. Peptides 2006, 27, 931-940.

10. Schneider, J. J.; Unholzer, A.; Schaller, M.; Schafer-Korting, M.; Korting, H. C. J. Mol. Med. 2005, 83, 587-595.

11. Landon, C.; Sodano, P.; Hetru, C.; Hoffmann, J.; Ptak, M. Protein. Sci. 1997, 6, 1878-1884.

12. Yamada, K.; Natori, S. Biochem. J. 1994, 3, 623-628.

13. Nygaard, M. K.; Andersen, A. S.; Kristensen, H. H.; Krogfelt, K. A.; Pojan, P.; Wimmer, R. J. Biomol. NMR 2012, 52, 277-282.

14. Hwang, J. S.; Kang, B. R.; Kim, S. R.; Yun, E. Y.; Park, K. H.; Jeon, J. P.; Nam, S. H.; Suh, H. W.; Hong, M. Y.; Kim, I. Int. J. Indust. Entomol. 2008, 17, 131-135.

15. Shin, S.; Kim, J. K.; Lee, J. Y.; Jung, K. W.; Hwang, J. S.; Lee, J.; Lee, D. G.; Kim, I.; Shin, S. Y.; Kim, Y. J. Pept. Sci. 2009, 15 559-568.

16. Lee, E.; Kim, J. K.; Shin, S.; Jeong, K. W.; Lee, J.; Lee, D. G.; Hwang, J. S.; Kim, Y. J. Pept. Sci. 2011, 17, 675-682.

17. Lee, E.; Kim, J. K.; Shin, S.; Jeong, K. W.; Shin, A.; Lee, J.; Lee, D. G.; Hwang, J. S.; Kim. Y. Biochim. Biophys. Acta 2013, 1828, 271-283.

18. Lee, J.; Hwang, J. S.; Hwang, I. S.; Cho, J.; Lee, E.; Kim, Y.; Lee, D. G. Free Radic. Biol. Med. 2012, 52, 2302-2311.

19. Hwang, J. S.; Lee, J.; Kim, Y. J.; Bang, H. S.; Yun, E. Y.; Kim, S. R.; Suh, H. J.; Kang, B. R.; Nam, S. H.; Jeong, J. P.; Kim, I.; Lee, D. G. Int. J. Pept. 2009, 2009, 1-5.

20. Lee, E.; Jeong, K. W.; Lee, J.; Shin, A.; Kim, J. K.; Lee, J.; Lee, D. G.; Kim, Y. BMB Reports 2013, 46, 282-287.

21. Jeong, K. W.; Shin, S.; Kim, J. K.; Kim, Y. Bull. Korean Chem. Soc. 2009, 30, 1839-1844.

22. Kim, J. K.; Lee, E.; Shin, S.; Jeong, K. W.; Lee, J. Y.; Bae, S. Y.; Kim, S. H.; Lee, J. Y.; Kim, S. R.; Hwang, J. S.; Kim, Y. J. Biol. Chem. 2011, 286, 41296-41311.

23. Hopp, T. P.; Woods, K. R. Proc. Natl. Acad. Sci. 1981, 78, 38243828.

24. Jeong, K. W.; Shin, S.; Kim, J. K.; Kim, Y. Bull. Korean Chem. Soc. 2011, 32, 2577-2583. 